\title{
A Strong Law of Large Numbers for Set-Valued Random Variables in $G_{\alpha}$ Space
}

\author{
Guan Li \\ College of Applied Sciences, Beijing University of Technology, Beijing, China \\ Email: guanli@bjut.edu.cn
}

Received 30 March 2015; accepted 23 June 2015; published 30 June 2015

\begin{abstract}
In this paper, we shall represent a strong law of large numbers (SLLN) for weighted sums of setvalued random variables in the sense of the Hausdorff metric $d_{H}$, based on the result of single-valued random variable obtained by Taylor [1].
\end{abstract}

Keywords

Set-Valued Random Variable, the Laws of Large Numbers, Hausdorff Metric

\section{Introduction}

We all know that the limit theories are important in probability and statistics. For single-valued case, many beautiful results for limit theory have been obtained. In [1], there are many results of laws of large numbers at different kinds of conditions and different kinds of spaces. With the development of set-valued random theory, the theory of set-valued random variables and their applications have become one of new and active branches in probability theory. And the theory of set-valued random variables has been developed quite extensively (cf. [2][7] etc.). In [1], Artstein and Vitale used an embedding theorem to prove a strong law of large numbers for independent and identically distributed set-valued random variables whose basic space is $\mathbb{R}^{d}$, and Hiai extended it to separable Banach space $\mathfrak{X}$ in [8]. Taylor and Inoue proved SLLN's for only independent case in Banach space in [7]. Many other authors such as Giné, Hahn and Zinn [9], Puri and Ralescu [10] discussed SLLN's under different settings for set-valued random variables where the underlying space is a separable Banach space.

In this paper, what we concerned is the SLLN of set-valued independent random variables in $G_{\alpha}$ space. Here the geometric conditions are imposed on the Banach spaces to obtain SLLN for set-valued random variables. The results are both the extension of the single-valued's case and the extension of the set-valued's case.

This paper is organized as follows. In Section 2, we shall briefly introduce some definitions and basic results of set-valued random variables. In Section 3, we shall prove a strong law of large numbers for set-valued independent random variables in $G_{\alpha}$ space.

\section{Preliminaries on Set-Valued Random Variables}

Throughout this paper, we assume that $(\Omega, \mathcal{A}, \mu)$ is a nonatomic complete probability space, $(\mathfrak{X},\|\cdot\|)$ is a real separable Banach space, $\mathbb{N}$ is the set of nature numbers, $\mathbf{K}(\mathfrak{X})$ is the family of all nonempty closed subsets 
of $\mathfrak{X}$, and $\mathbf{K}_{b c}(\mathfrak{X})$ is the family of all nonempty bounded closed convex subsets of $\mathfrak{X}$.

Let $A$ and $B$ be two nonempty subsets of $\mathfrak{X}$ and let $\lambda \in \mathbb{R}$, the set of all real numbers. We define addition and scalar multiplication as

$$
\begin{gathered}
A+B=\{a+b: a \in A, b \in B\}, \\
\lambda A=\{\lambda a: a \in A\} .
\end{gathered}
$$

The Hausdorff metric on $\mathbf{K}(\mathfrak{X})$ is defined by

$$
d_{H}(A, B)=\max \left\{\operatorname{supinf}_{a \in A}\|a-b\| \operatorname{supinf}_{b \in B}\|a-b\|\right\},
$$

for $A, B \in \mathbf{K}(\mathfrak{X})$. For an $A$ in $\mathbf{K}(\mathfrak{X})$, let $\|A\|_{\mathbf{K}}=d_{H}(\{0\}, A)$. The metric space $\left(\mathbf{K}_{b}(\mathfrak{X}), d_{H}\right)$ is complete, and $\mathbf{K}_{b c}(\mathfrak{X})$ is a closed subset of $\left(\mathbf{K}_{b}(\mathfrak{X}), d_{H}\right)$ (cf. [6], Theorems 1.1.2 and 1.1.3). For more general hyperspaces, more topological properties of hyperspaces, readers may refer to a good book [11].

For each $A \in \mathbf{K}(\mathfrak{X})$, define the support function by

$$
s\left(x^{*}, A\right)=\sup _{a \in A}<x^{*}, a>, x^{*} \in \mathfrak{X}^{*},
$$

where $\mathfrak{X}^{*}$ is the dual space of $\mathfrak{X}$.

Let $\mathbf{S}^{*}$ denote the unit sphere of $\mathfrak{X}^{*}, C\left(\mathbf{S}^{*}\right)$ the all continuous functions of $\mathbf{S}^{*}$, and the norm is defined as $\|v\|_{C}=\sup _{x}{ }^{*} \in S^{*}$

The following is the equivalent definition of Hausdorff metric.

For each $A, B \in \mathbf{K}_{b c}(\mathfrak{X})$,

$$
d_{H}(A, B)=\sup \left\{\left|s\left(x^{*}, A\right)-s\left(x^{*}, A\right)\right|: x^{*} \in S^{*}\right\} .
$$

A set-valued mapping $F: \Omega \rightarrow \mathbf{K}(\mathfrak{X})$ is called a set-valued random variable (or a random set, or a multifunction) if, for each open subset $O$ of $\mathfrak{X}, F^{-1}(O)=\{\omega \in \Omega: F(\omega) \cap O \neq \varnothing\} \in A$.

For each set-valued random variable $F$, the expectation of $F$, denoted by $E[F]$, is defined as

$$
E[F]=\left\{\int_{\Omega} f d \mu: f \in S_{F}\right\},
$$

where $\int_{\Omega} f d \mu$ is the usual Bochner integral in $L^{1}[\Omega, \mathfrak{X}]$, the family of integrable $\mathfrak{X}$-valued random variables, and $S_{F}=\left\{f \in L^{1}[\Omega ; X]: f(\omega) \in F(\omega)\right.$, a.e. $\left.(\mu)\right\}$. This integral was first introduced by Aumann [3], called Aumann integral in literature.

\section{Main Results}

In this section, we will give the limit theorems for independent set-valued random variables in $G_{\alpha}$ space. The following definition and lemma are from [1], which will be used later.

Definition 3.1 A Banach space $\mathfrak{X}$ is said to satisfy the condition $G_{\alpha}$ for some $\alpha, 0<\alpha \leq 1$, if there exists a mapping $G: \mathfrak{X} \rightarrow \mathfrak{X}^{*}$ such that

(i) $\|G(x)\|=\|x\|^{\alpha}$;

(ii) $G(x) x=\|x\|^{1+\alpha}$;

(iii) $\|G(x)-G(y)\| \leq A\|x-y\|^{\alpha}$ for all $x, y \in \mathfrak{X}$ and some positive constant $A$.

Note that Hilbert spaces are $G_{1}$ with constant $A=1$ and identity mapping $G$.

Lemma 3.1 Let $\mathfrak{X}$ be a separable Banach space which is $G_{\alpha}$ for some $0<\alpha \leq 1$ and let $\left\{V_{1}, V_{2}, \cdots, V_{n}\right\}$ be single-valued independent random elements in $\mathfrak{X}$ such that $E\left[V_{k}\right]=0$ and $E\left[\left\|V_{k}\right\|^{1+\alpha}\right]<\infty$ for each $k=1,2, \cdots, n$. then

$$
E\left[\left\|V_{1}+\cdots+V_{n}\right\|^{1+\alpha}\right] \leq A \sum_{k=1}^{n} E\left[\left\|V_{k}\right\|^{1+\alpha}\right]
$$


where $A$ is the positive constant in (iii).

Theorem 3.1 Let $\mathfrak{X}$ be a separable Banach space which is $G_{\alpha}$ for some $0<\alpha \leq 1$. Let $\left\{F_{n}: n \geq 1\right\}$ be a sequence of independent set-valued random variables in $\mathbf{K}_{b c}(\mathfrak{X})$, such that $E\left[F_{n}\right]=\{0\}$ for each $n$. If

$$
\sum_{j=1}^{\infty} E\left[\left\|F_{j}\right\|_{\mathbf{K}}\right]^{1+\alpha}<\infty
$$

where $\phi_{0}(t)=t^{1+\alpha}$ for $0 \leq t \leq 1$ and $\phi_{0}(t)=t$ for $t \geq 1$, then $\sum_{j=1}^{\infty} F_{j}$ converges with probability 1 in the sense of $d_{H}$.

Proof. Define

$$
U_{j}=F_{j} I_{\left\{\left\|F_{j}\right\|_{K} \leq 1\right\}} \text { and } \quad W_{j}=F_{j} I_{\left\{\left\|F_{j}\right\|_{K}>1\right\}} \text {. }
$$

Note that $F_{j}=U_{j}+W_{j}$ for each $j$ and that both $\left\{U_{j}: j \geq 1\right\}$ and $\left\{W_{j}: j \geq 1\right\}$ are independent sequences of set-valued random variables. Next, for each $m$ and $n$

$$
E\left[\left\|\sum_{j=n}^{m} W_{j}\right\|_{\mathbf{K}}\right] \leq \sum_{j=n}^{m} E\left[\left\|W_{j}\right\|_{\mathbf{K}}\right] \leq \sum_{j=n}^{m} E\left[\phi_{0}\left(\left\|F_{j}\right\|_{\mathbf{K}}\right)\right] .
$$

That means $\left\{E\left[\left\|\sum_{j=1}^{m} W_{j}\right\|_{\mathbf{K}}\right]: m \geq 1\right\}$ is a Cauchy sequence and hence

$$
E\left[\left\|\sum_{j=1}^{m} W_{j}\right\|_{\mathbf{K}}\right] \text { converges }
$$

as $m \rightarrow \infty$. Since convergence in the mean implied convergence in probability, Ito and Nisio's result in [12] for independent random elements(rf. Section 4.5) provides that

$$
\left\|\sum_{j=1}^{\infty} W_{j}\right\|_{K} \text { converges in probability } 1 \text { as } n \rightarrow \infty .
$$

Then for $n, m \geq 1, m>n$, by triangular inequality we have

$$
\begin{aligned}
& d_{H}\left(\sum_{j=1}^{n} W_{j}, \sum_{j=1}^{m} W_{j}\right)=d_{H}\left(\sum_{j=1}^{n} W_{j}, \sum_{j=1}^{n} W_{j}+\sum_{j=n+1}^{m} W_{j}\right) \\
& \leq d_{H}\left(\{0\}, \sum_{j=n+1}^{m} W_{j}\right)=\left\|\sum_{j=n+1}^{m} W_{j}\right\|_{\mathbf{K}} \rightarrow 0, \text { a.e. as } n, m \rightarrow \infty .
\end{aligned}
$$

By the completeness of $\left(\mathbf{K}_{b}(\mathfrak{X}), d_{H}\right)$, we can have $\sum_{j=1}^{n} W_{j}$ converges almost everywhere in the sense of $d_{H}$. Since by equivalent definition of Hausdorff metric, we have

$$
\begin{aligned}
E\left[\left\|\sum_{j=n}^{m} U_{j}\right\|_{\mathbf{K}}^{1+\alpha}\right] & =E\left[d_{H}\left(\sum_{j=n}^{m} U_{j},\{0\}\right)\right]^{1+\alpha} \\
& =E\left[\sup _{x^{*} \in S^{*}}\left|s\left(x^{*}, \sum_{j=n}^{m} U_{j}\right)\right|\right]^{1+\alpha} .
\end{aligned}
$$

For any fixed $n, m$, there exists a sequence $x_{k}^{*} \in S^{*}$, such that

$$
\lim _{k \rightarrow \infty}\left|s\left(x_{k}^{*}, \sum_{j=n}^{m} U_{j}\right)\right|=\sup _{x^{*} \in S^{*}}\left|s\left(x^{*}, \sum_{j=n}^{m} U_{j}\right)\right| .
$$

Then by dominated convergence theorem, Minkowski inequality and Lemma 3.1, we have 


$$
\begin{aligned}
& E\left[\left\|\sum_{j=n}^{m} U_{j}\right\|_{\mathbf{K}}^{1+\alpha}\right]=E\left[\lim _{k \rightarrow \infty}\left|s\left(x_{k}^{*}, \sum_{j=n}^{m} U_{j}\right)\right|^{1+\alpha}\right]=\lim _{k \rightarrow \infty} E\left[\left|s\left(x_{k}^{*}, \sum_{j=n}^{m} U_{j}\right)\right|^{1+\alpha}\right] \\
& \leq \lim _{k \rightarrow \infty} E\left[\left|s\left(x_{k}^{*}, \sum_{j=n}^{m} U_{j}\right)-E\left[s\left(x_{k}^{*}, \sum_{j=n}^{m} U_{j}\right)\right]\right|+\left|E\left[s\left(x_{k}^{*}, \sum_{j=n}^{m} U_{j}\right)\right]\right|\right]^{1+\alpha} \\
& =\lim _{k \rightarrow \infty} E\left[\left|s\left(x_{k}^{*}, \sum_{j=n}^{m} U_{j}\right)-E\left[s\left(x_{k}^{*}, \sum_{j=n}^{m} U_{j}\right)\right]\right|+\left|E\left[s\left(x_{k}^{*}, \sum_{j=n}^{m} U_{j}\right)\right]\right|\right]^{1+\alpha} \\
& \leq 2^{1+\alpha}\left\{A \lim _{k \rightarrow \infty} \sum_{j=n}^{m} E\left[\left|s\left(x_{k}^{*}, U_{j}\right)-s\left(x_{k}^{*}, E\left[U_{j}\right]\right)\right|^{1+\alpha}\right]+\lim _{k \rightarrow \infty}\left[\sum_{j=n}^{m} E\left[\left|s\left(x_{k}^{*}, W_{j}\right)\right|\right]\right]^{1+\alpha}\right\} \\
& \left.\leq 2^{1+\alpha}\left\{\left.A 2^{2+\alpha} \sum_{j=n}^{m} E\left[\sup _{x^{*} \in S^{*}} \mid s\left(x^{*}, U_{j}\right)\right)\right|^{1+\alpha}\right]+\left[\sum_{j=n}^{m} E\left[\sup _{x^{*} \in S^{*}}\left|s\left(x^{*}, W_{j}\right)\right|\right]\right]^{1+\alpha}\right\} \\
& \leq 2^{1+\alpha}\left\{A 2^{2+\alpha} \sum_{j=n}^{m} E\left[\phi_{0}\left(\left\|F_{j}\right\|_{\mathbf{K}}\right)\right]+\left[\sum_{j=n}^{m} E\left[\phi_{0}\left(\left\|F_{j}\right\|_{\mathbf{K}}\right)\right]\right]^{1+\alpha}\right\}
\end{aligned}
$$

for each $n$ and $m$. Thus, $\left\{E\left[\left\|\sum_{j=1}^{m} U_{j}\right\|_{\mathbf{K}}\right]: m \geq 1\right\}$ is a Cauchy sequence, and hence converges. Hence, by the similar way as above to prove $\sum_{j=1}^{\infty} W_{j}$ converges with probability one in the sense of $d_{H}$. We also can prove that

$$
\sum_{j=1}^{\infty} U_{j} \quad \text { converges }
$$

with probability one in the sense of $d_{H}$. The result was proved.

From theorem 3.1, we can easily obtain the following corollary.

Corollary 3.2 Let $\mathfrak{X}$ be a separable Banach space which is $G_{\alpha}$ for some $0<\alpha \leq 1$. Let $\left\{F_{n}\right\}$ be a sequence of independent set-valued random variables in $\mathbf{K}_{b}(\mathfrak{X})$ such that $E\left[F_{n}\right]=\{0\}$ for each $n$. If $\phi_{n}: R^{+} \rightarrow R^{+}, n=1,2, \cdots$, are continuous and such that $\frac{\phi_{n}(t)}{t}$ and $\frac{t^{1+\alpha}}{\phi_{n}(t)}$ are non-decreasing, then for each $\alpha_{n} \subset R^{+}$the convergence of

$$
\sum_{n=1}^{\infty} \frac{E\left[\phi_{n}\left(\left\|F_{n}\right\|_{\mathbf{K}}\right)\right]}{\phi_{n}\left(\alpha_{n}\right)}
$$

implies that

$$
\sum_{n=1}^{\infty} \frac{F_{n}}{\alpha_{n}}
$$

converges with probability one in the sense of $d_{H}$.

Proof. Let

$$
U_{j}=\frac{F_{j}}{\alpha_{j}} I_{\left\{\left\|F_{j}\right\|_{\mathbf{K}} \leq \alpha_{j}\right\}} \quad \text { and } \quad W_{j}=\frac{F_{j}}{\alpha_{j}} I_{\left\{\left\|F_{j}\right\|_{\mathbf{K}}>\alpha_{j}\right\}} .
$$

If $\left\|F_{n}\right\|_{K}>\alpha_{n}$, by the non-decreasing property of $\frac{\phi_{n}(t)}{t}$, we have

$$
\frac{\phi_{n}\left(\alpha_{n}\right)}{\alpha_{n}} \leq \frac{\phi_{n}\left(\left\|F_{n}\right\|_{\mathbf{K}}\right)}{\left\|F_{n}\right\|_{\mathbf{K}}} \text {. }
$$

That is 


$$
\frac{\left\|F_{n}\right\|_{\mathbf{K}}}{\alpha_{n}} \leq \frac{\phi_{n}\left(\left\|F_{n}\right\|_{\mathbf{K}}\right)}{\phi_{n}\left(\alpha_{n}\right)}
$$

If $\left\|F_{n}\right\|_{K} \leq \alpha_{n}$, by the non-decreasing property of $\frac{t^{1+\alpha}}{\phi_{n}(t)}$, we have

$$
\frac{\left\|F_{n}\right\|_{\mathrm{K}}^{1+\alpha}}{\phi_{n}\left(\left\|F_{n}\right\|_{\mathrm{K}}\right)} \leq \frac{\alpha_{n}^{1+\alpha}}{\phi_{n}\left(\alpha_{n}\right)} \text {. }
$$

That is

$$
\frac{\left\|F_{n}\right\|_{\mathbf{K}}^{1+\alpha}}{\alpha_{n}^{1+\alpha}} \leq \frac{\phi_{n}\left(\left\|F_{n}\right\|_{\mathbf{K}}\right)}{\phi_{n}\left(\alpha_{n}\right)} .
$$

Then as the similar proof of theorem 3.1, we can prove both $\sum_{j=1}^{\infty} U_{j}$ and $\sum_{j=1}^{\infty} W_{j}$ converges with probability one, and the result was obtained.

\section{Acknowledgements}

The research was supported by NSFC(11301015, 11401016, 11171010), BJNS (1132008).

\section{References}

[1] Taylor, R.L. (1978) Lecture Notes in Mathematics. Springer-Verlag.

[2] Artstein, Z. and Vitale, R.A. (1975) A Strong Law of Large Numbers for Random Compact Sets. Ann. Probab., 3, 879-882. http://dx.doi.org/10.1214/aop/1176996275

[3] Aumann, R. (1965) Integrals of Set Valued Functions. J. Math. Anal. Appl., 12, 1-12. http://dx.doi.org/10.1016/0022-247X(65)90049-1

[4] Hiai, F. and Umegaki, H. (1977) Integrals, Conditional Expectations and Martingales of Multivalued Functions. J. Multivar. Anal., 7, 149-182. http://dx.doi.org/10.1016/0047-259X(77)90037-9

[5] Jung, E.J. and Kim, J.H. (2003) On Set-Valued Stochastic Integrals. Stoch. Anal. Appl., 21, 401-418. http://dx.doi.org/10.1081/SAP-120019292

[6] Li, S., Ogura, Y. and Kreinovich, V. (2002) Limit Theorems and Applications of Set-Valued and Fuzzy Set-Valued Random Variables. Kluwer Academic Publishers, Dordrecht. http://dx.doi.org/10.1007/978-94-015-9932-0

[7] Taylor, R.L. and Inoue, H. (1985) A Strong Law of Large Numbers for Random Sets in Banach Spaces. Bull. Instit. Math. Academia Sinica, 13, 403-409.

[8] Hiai, F. (1984) Strong Laws of Large Numbers for Multivalued Random Variables, Multifunctions and Integrands. In: Salinetti, G., Ed., Lecture Notes in Math., Springer, Berlin, 1091, 160-172.

[9] Giné, E., Hahn, G. and Zinn, J. (1983) Limit Theorems for Random Sets: An Application of Probability in Banach Space Results. Lect. Notes in Math., 990, 112-135. http://dx.doi.org/10.1007/bfb0064267

[10] Puri, M.L. and Ralescu, D.A. (1983) Strong Law of Large Numbers for Banach Space Valued Random Sets. Ann. Probab., 11, 222-224. http://dx.doi.org/10.1214/aop/1176993671

[11] Beer, G. (1993) Topologies on Closed and Closed Convex Sets, Mathematics and Its Applications. Kluwer Academic Publishers, Dordrecht, Holland. http://dx.doi.org/10.1007/978-94-015-8149-3

[12] Itô, K. and Nisio, M. (1968) On the Convergence of Sums of Independent Banach Space Valued Random Variables. Osaka J. Math., 5, 35-48. 